\title{
Tailoring of optical and electrical properties of PMMA by incorporation of Ag nanoparticles
}

\author{
ALISHA GOYAL ${ }^{1}$, ANNU SHARMA ${ }^{1, *}$, ISHA SAINI ${ }^{1}$, NAVNEET CHANDAK ${ }^{2}$ and \\ PAWAN SHARMA ${ }^{2}$ \\ ${ }^{1}$ Department of Physics, Kurukshetra University, Kurukshetra, India \\ ${ }^{2}$ Department of Chemistry, Kurukshetra University, Kurukshetra, India \\ *Author for correspondence (talk2annu@ gmail.com)
}

MS received 19 May 2016; accepted 3 October 2016; published online 25 July 2017

\begin{abstract}
Silver-poly(methyl methacrylate) (Ag-PMMA) nanocomposite films were prepared via ex situ chemical route by employing sodium borohydride $\left(\mathrm{NaBH}_{4}\right)$ as a reducing agent. In this study, PVP-stabilized Ag nanoparticles were prepared and mixed with PMMA solution. Optical and structural characterizations of resulting nanocomposite films were performed using UV-visible spectroscopy, transmission electron microscopy (TEM) and scanning electron microscopy (SEM). Characteristic surface plasmon resonance (SPR) peak of Ag nanoparticles was observed at about $3.04 \mathrm{eV}(408 \mathrm{~nm})$ in absorption spectra of Ag-PMMA nanocomposite films. TEM micrograph revealed that the spherical Ag nanoparticles with an average diameter of 5.4 $\pm 2.5 \mathrm{~nm}$ are embedded in PMMA. In Raman spectra, besides shifting of vibrational bands, enhancement in intensity of Raman signal with incorporation of Ag nanoparticles was observed. Current $(I)$-voltage $(V)$ measurements revealed that conductivity of PMMA increased with increasing concentration of Ag nanoparticles. Analysis of $I-V$ data further disclosed that at voltage $<2 \mathrm{~V}$, ohmic conduction mechanism is the dominant mechanism, while at voltage $>2 \mathrm{~V}$ Poole-Frenkel is the dominant conduction mechanism. Urbach's energy, the measure of disorder, increased from $0.40 \mathrm{eV}$ for PMMA to $1.11 \mathrm{eV}$ for Ag-PMMA nanocomposite films containing $0.039 \mathrm{wt} \%$ of Ag nanoparticles.
\end{abstract}

Keywords. PMMA; Ag nanoparticles; Urbach's energy; nanocomposite; surface-enhanced Raman scattering.

\section{Introduction}

Polymers are routinely being used in number of technological applications due to their exceptional properties such as low cost, light weight, stiffness, reduced cost and corrosion resistance. However, their usages have always been limited due to their redundant properties such as poor optical, electrical, barrier, thermal and mechanical properties. Incorporation of nanoparticles into polymer matrix to form nanocomposites is a unique way of improving their properties [1]. Embedded nanoparticles contribute towards the enhancement in electrical, optical and dielectric properties of the polymer and the polymer matrix in turn protects nanoparticles from agglomeration and environmental and chemical exposures. Moreover, the distinctive properties of polymers such as energy gap, refractive index, dielectric constants can be tuned by varying the shape, size and quantity of incorporated metal nanoparticles [2-5]. Such nanocomposites have potential applications in optics [1], electronics [1], photonics [3] and medical science $[2,6]$.

Poly(methyl methacrylate) (PMMA) is an acrylic polymer with distinct properties such as high transmission of visible light, environmental stability and durability leading to its wide spread applications in wave guides, optical devices, hard contact lenses, luminous ceilings, aircraft windows, etc. Although
PMMA has distinct properties, it has some inherent drawbacks, such as low tensile strength, low absorption of visible light, low electrical conductivity and small positive temperature coefficient of resistance $(\sim 1-3$ orders magnitude change in resistivity) [4], which limits its use as temperature sensors and in other optical, medical, industrial applications. Several techniques like ion irradiation, ion implantation, encapsulating of nanofillers into the PMMA matrix are routinely being used to improve the properties of PMMA [5,7-9].

Among various metal nanoparticles, noble metals like $\mathrm{Cu}$, $\mathrm{Ag}$ and $\mathrm{Au}$ exhibit a strong surface plasmon resonance (SPR) peak in visible region due to the collective oscillation of the valence electrons [5]. The SPR peak can be tuned by changing particle size, shape, as well as the surrounding dielectric medium $[3,5]$. This tunability makes them interesting candidates for potential applications in surface-enhanced spectroscopy [10], optical filters [1], plasmonic devices, sensors, photovoltaic devices and imaging [11]. Ag nanoparticles exhibit higher efficiency of plasmon excitation which in turn leads to enhanced properties including catalysis [6], magnetic and optical polarizabilities [1], electrical conductivity [1] and antimicrobial activity [6]. Thus, encapsulation of Ag nanoparticles in polymer host matrix like PMMA adds together the exotic properties of Ag nanoparticles and PMMA and resulting in Ag-PMMA nanocomposites, which could 
find applications in static-discharge housing and packaging for electronics; electromagnetic interference shielding; and lightweight, flexible conductors for electrodes, circuits, displays and sensors. Moreover optical, electrical and structural properties of PMMA can be tailored by controlling the size and shape of $\mathrm{Ag}$ nanoparticles rather than by altering the composition. Therefore, fabrication and study of Ag-PMMA nanocomposites is expected to be an advert area of research, for their superior properties.

Ag-PMMA nanocomposite films can be fabricated by several methods such as ion implantation [7], physical vapour deposition (PVD) [8], photolysis [9] and in situ chemical route [5], etc. However, synthesis of Ag-PMMA nanocomposite films via ex situ chemical route has rarely been discussed. Through ex situ chemical technique, pre-synthesized nanoparticles with controlled size and shape can be incorporated into the polymer host matrices. In literature, there are hardly any reports on optical, structural and electrical properties of AgPMMA nanocomposite synthesized by ex situ chemical route. In the present work, ex situ chemical synthesis technique has been chosen to fabricate Ag-PMMA nanocomposite films.

In the present work, pre-synthesized Ag nanoparticles were dispersed into PMMA with the help of polyvinylpyrrolidone (PVP) as a capping agent and ethanol as a binding agent to fabricate Ag-PMMA nanocomposite films. The present study has mainly focussed on modification of optical, structural and electrical properties of PMMA by embedding Ag nanoparticles. The simplicity and affordability of synthesis of Ag-PMMA nanocomposites by this method, render them one of the most exceptional and market-competitive materials for applications in photovoltaics, light emitting diodes (LED), photodetectors, lasers, sensors, etc.

\section{Experimental}

\subsection{Materials}

Ag-PMMA nanocomposite films were prepared by an ex situ chemical route as discussed elsewhere [12]. In the present work, all chemicals and reagents used were of analytical grade and were used without further purification. The thickness of Ag-PMMA nanocomposite films was about $25 \mu \mathrm{m}$.

\subsection{Instruments}

The optical properties of PMMA and Ag-PMMA nanocomposite films were analysed using a Shimadzu Double Beam Double Monochromator Spectrophotometer (UV-2550), equipped with an integrating sphere assembly ISR-240A in the wavelength range of 190-900 $\mathrm{nm}$ with a resolution of $0.5 \mathrm{~nm}$. The absorption and transmission spectra were recorded by keeping air as reference, whereas for recording reflection spectra, $\mathrm{BaSO}_{4}$ was taken as a reference. The morphology of Ag nanoparticles embedded in PMMA has been studied using TEM (Hitachi H-7500). For TEM images, a small quantity of nanocomposite film was added to methanol and sonicated for $20 \mathrm{~min}$ via a sonication bath, and then a few drops of the nanocomposite film solution were deposited on a carbon-coated copper grid. The grid was dried for 5-10 min, so that the excess solvent gets evaporated. Topography and morphology of Ag-PMMA nanocomposite films were further confirmed using SEM (JEOL JSM 6390LV). To ascertain the interaction of Ag nanoparticles with PMMA, Raman spectrum of PMMA and Ag-PMMA nanocomposite films was recorded using Dilor Raman Spectrometer having 532 $\mathrm{nm}$ wavelength laser with a resolution of 3-5 nm. Electrical measurements of PMMA and Ag-PMMA nanocomposite films were carried out using the Keithley 6517A electrometer attached with Keithley 8009 resistivity test fixture. For $I-V$ measurements, Ag-PMMA nanocomposite films of $5 \mathrm{~cm}$ diameter were laid between the resistivity test fixtures and a testing voltage of $100 \mathrm{~V}$ was applied.

\section{Results and discussion}

Mie (1908) elucidated the interaction of electromagnetic radiation with small metal nanoparticles. According to Mie's theory, for a spherical metal nanoparticle with size much smaller than the wavelength of incident light $(2 r \ll \lambda)$, optical extinction cross-section $\left(\sigma_{\text {ext }}\right)$ can be given by the following equation (quasi-static or dipole approximation):

$$
\sigma_{\text {ext }}(\omega)=\frac{9 \omega \varepsilon_{\mathrm{m}}(\omega)^{3 / 2} V \varepsilon_{2}(\omega)}{c\left\{\left[\varepsilon_{1}(\omega)+2 \varepsilon_{\mathrm{m}}\right]^{2}+\varepsilon_{2}(\omega)^{2}\right.},
$$

where $V$ is the particle volume, $\omega$ the angular frequency of incident light, $c$ the velocity of light. $\varepsilon_{\mathrm{m}}$ is the dielectric constant of the surrounding medium and $\varepsilon(\omega)=\varepsilon_{1}(\omega)+i \varepsilon_{2}(\omega)$ is the complex dielectric function of metal nanoparticle. In the presence of electric field of incident light, metal nanoparticle gets polarized and behaves as a dipole. When $\varepsilon_{1}=-2 \varepsilon_{\mathrm{m}}$, maximum absorption of energy by metal nanoparticle and SPR occur $[13,14]$. The real part $\varepsilon_{1}(\omega)$ of the dielectric function of the metal determines the SPR position and the imaginary part $\varepsilon_{2}(\omega)$ determines the bandwidth. Thus, the energy of the SPR depends on both the free electron density and the dielectric medium surrounding the nanoparticle. Variation in these parameters alters the position as well as the intensity of SPR band. Noble metal nanoparticles, like gold, silver and copper show strong SPR bands in the visible region. Figure 1 displays the UV-visible absorption spectra of PMMA and Ag-PMMA nanocomposite films in energy range of 1.5-5.0 eV. Two peaks at 4.23 and $3.56 \mathrm{eV}$ in the absorption spectra of PMMA matrix can be attributed to the $n \rightarrow \pi^{*}$ transition of $\mathrm{C}=\mathrm{O}$ and $\mathrm{C}-\mathrm{O}$ bonds present in PMMA [15]. Appearance of broad absorption band also known as SPR band is observed at about $3.04 \mathrm{eV}$ (408 nm) in Ag-PMMA nanocomposite films (figure 1 (curves $b-d)$ ). This spectral band is ascribed to the collective oscillations of the free electrons of Ag nanoparticles stimulated by incident light. 


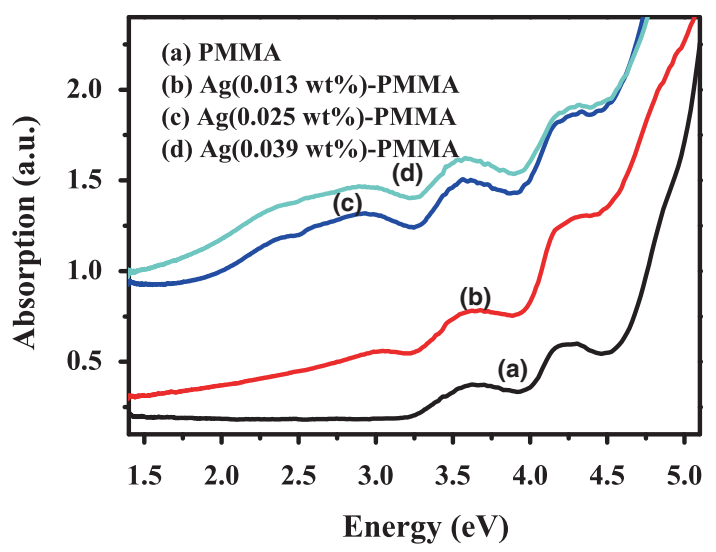

Figure 1. Absorption spectra of (a) PMMA and Ag-PMMA nanocomposite films containing (b) $0.013 \mathrm{wt} \%$, (c) $0.025 \mathrm{wt} \%$ and (d) $0.039 \mathrm{wt} \% \mathrm{Ag}$ nanoparticles.

SPR peak is sensitive to the microstructure of the sample, i.e., size and shape of the dispersed metal nanoparticles, concentration of metal phase and dielectric constant of host medium [5]. The intensity of SPR band increases with increasing concentration of $\mathrm{Ag}$ nanoparticles in PMMA and it also shifts towards lower energy (red-shift). From the absorption spectra, the size of Ag nanoparticles was calculated assuming the free particle behaviour of the conduction electrons, using the relation [16]

$$
d=\frac{h v_{\mathrm{f}}}{\pi \Delta E_{1 / 2}},
$$

where $d$ is the diameter of the $\mathrm{Ag}$ nanoparticle, $v_{\mathrm{f}}(1.39 \times$ $10^{6} \mathrm{~m} \mathrm{~s}^{-1}$ ) the Fermi velocity of electrons in bulk silver, $h$ the Planck's constant and $\Delta E_{1 / 2}$ the FWHM of the absorption band. The above equation is valid only for the $\mathrm{Ag}$ nanoparticles having dimensions smaller than mean free path of the electrons in bulk Ag metal $(52 \mathrm{~nm})$. The diameter of $\mathrm{Ag}$ nanoparticles from absorption spectra is about $5 \mathrm{~nm}$.

Theoretical estimation of SPR peak position of isolated Ag nanoparticles of diameter $5 \mathrm{~nm}$ embedded in PMMA matrix were carried out on the basis of classical Mie scattering theory [14] using 'Mieplot 4211' software. Figure 2 presents typical simulated absorption spectra of the isolated Ag nanoparticle (when diameter is $5 \mathrm{~nm}$ ) as a function of wavelength and the SPR peak position recorded experimentally. From this figure, it is clear that spherical Ag nanoparticles of size $5 \mathrm{~nm}$ embedded in PMMA matrix showed an SPR peak at $408 \mathrm{~nm}$.

Thus, we can deduce that for small spherical nanoparticles, the Mie theory is an efficient model to describe the optical response. However, when the particle size increases, the quasi-static approximation is no longer valid because the electrons do not experience a homogenous electric field, as a result, a red-shift of the plasmon resonance wavelength is observed for larger nanoparticles [5,13,14,17].

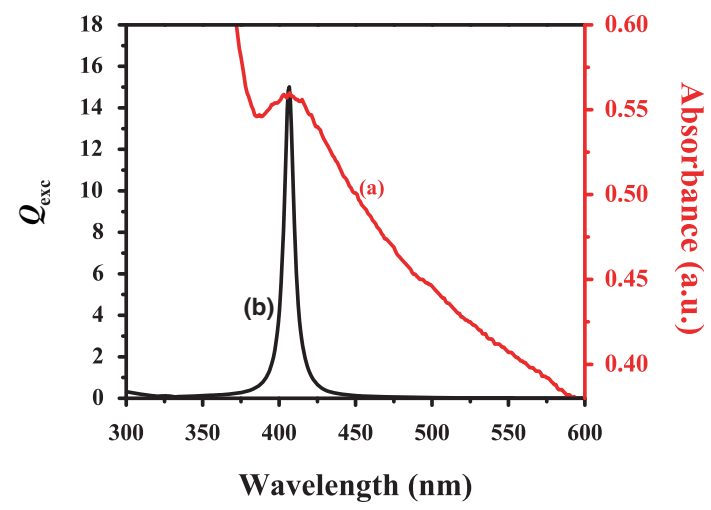

Figure 2. UV-visible absorption spectra of Ag-PMMA nanocomposite: (a) experimentally and (b) theoretically measured using Mie theory model.

Additionally, radiative losses increase when the particle size gets larger, which broadens the plasmon band width and reduces the intensity. Further, when the dielectric medium surrounding the nanoparticles changes, the condition for the resonance is altered. An increase in the dielectric function of the surrounding matrix gives rise to an increased screening of the surface charges, which weakens the restoring force on the electrons. As a result, less energy is required to excite the electrons which red-shifts the plasmon resonance frequency [17]. Thus, red-shift observed in SPR peak position with increasing concentration of $\mathrm{Ag}$ nanoparticles in PMMA matrix may be attributed to the increase in the particle size or wider size distribution of Ag nanoparticles and may be due to the interaction of Ag nanoparticles with PMMA.

Figure 2 (curve a) also shows that experimentally measured SPR peak is broadened when compared to the theoretically calculated peak. The broadening of the SPR peak may be due to the interaction of Ag nanoparticles with PMMA matrix and/or due to the wider size distribution of prepared $\mathrm{Ag}$ nanoparticles, while the prediction of the Mie theory is based on the fixed value of the particle diameter. Good agreement between theoretically estimated and experimentally measured values of SPR peak position was observed.

The shape, size and distribution of Ag nanoparticles in PMMA were investigated at room temperature using transmission electron microscopy (TEM). The average diameter for spherical Ag nanoparticles in Ag-PMMA nanocomposite containing $0.039 \mathrm{wt} \% \mathrm{Ag}$ nanoparticles was found to be $5.4 \pm 2.5 \mathrm{~nm}$, which is consistent with the result obtained from UV-visible spectrum. Figure 3 shows the typical TEM image and the corresponding particle size distribution (inset of figure 3 ) of the prepared sample. It is clearly observable from figure 3 that $\mathrm{Ag}$ nanoparticles are well distributed within PMMA.

The morphology and distribution of Ag nanoparticles in PMMA was further examined using scanning electron microscopy (SEM). Figure 4 depicts the SEM image of 


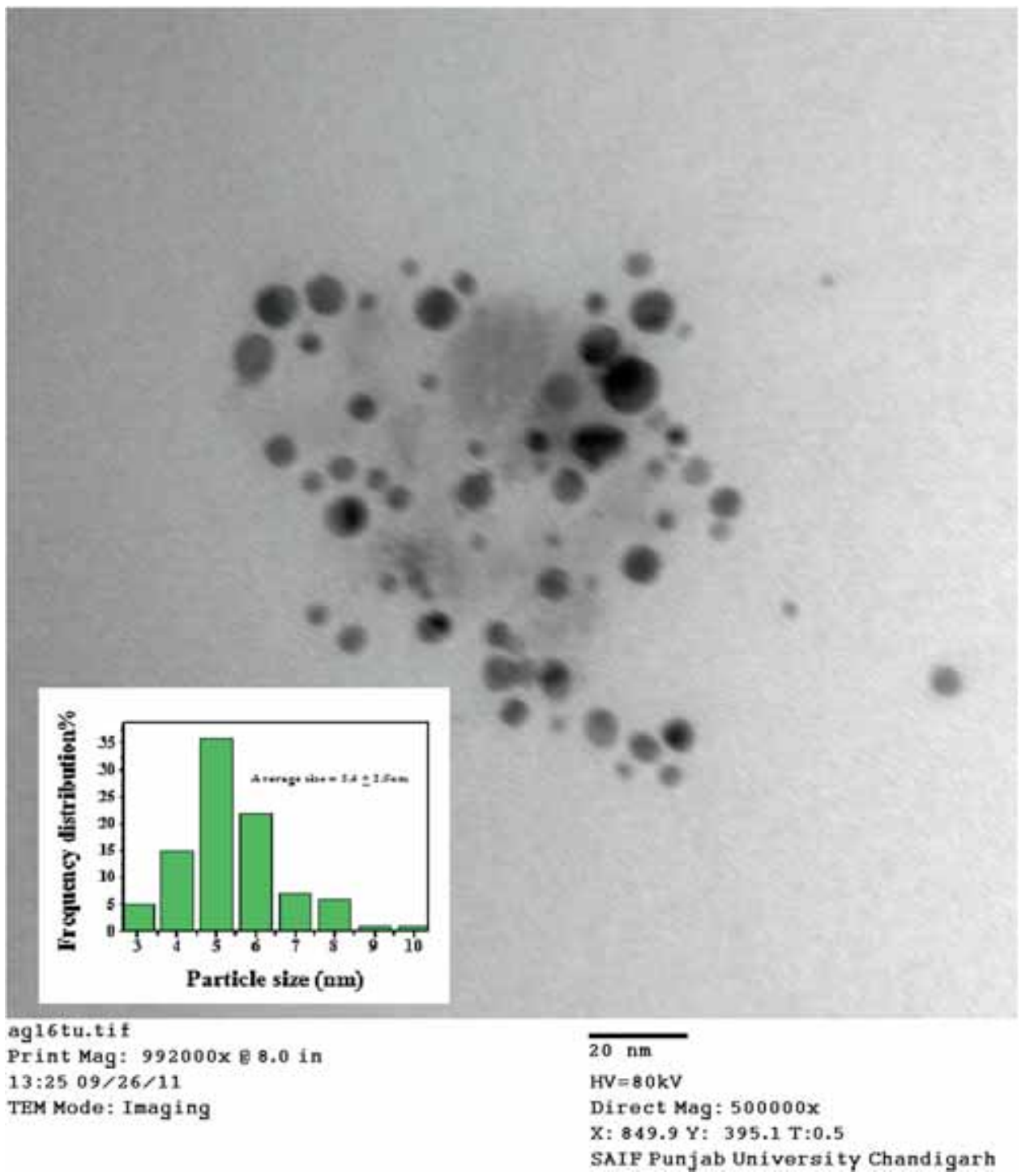

Figure 3. TEM image of Ag-PMMA incorporated with 0.039 wt $\%$ Ag nanoparticles and corresponding particle size distribution (inset).

Ag-PMMA nanocomposite film containing $0.039 \mathrm{wt} \% \mathrm{Ag}$ nanoparticles. It is seen from the micrograph that $\mathrm{Ag}$ nanoparticles in PMMA are almost spherical and have non-uniform size distribution. SEM micrograph further reveals that $\mathrm{Ag}$ nanoparticles are uniformly dispersed in PMMA. These results are in good agreement with those obtained by using TEM.

Figure 5 (curve a) depicts Raman spectra of PMMA and Ag-PMMA nanocomposite films. In Raman spectra of PMMA, main band at $600 \mathrm{~cm}^{-1}$ is assigned to $(\mathrm{C}-\mathrm{C}-\mathrm{O})$ and (C-COO) stretches, at $807 \mathrm{~cm}^{-1}$ is assigned to $(\mathrm{C}-\mathrm{O}-\mathrm{C})$ stretch, at $1450 \mathrm{~cm}^{-1}$ is assigned to $(\mathrm{C}-\mathrm{H})$ in plane bending and at $1729 \mathrm{~cm}^{-1}$ for $(\mathrm{C}=\mathrm{O})$ stretch. The most prominent band observed at $2957 \mathrm{~cm}^{-1}$ is due to the $(\mathrm{C}-\mathrm{H})$ stretching vibration. These vibrational bands ascertained the monomer structure of PMMA, which is in close agreement with those reported in literature $[18,19]$.

After addition of Ag nanoparticles in PMMA, besides enhancement in the Raman intensity and shifting of bands to low wavenumber side (figure 5 (curve b)), some new peaks were also observed. Irradiation of Ag-PMMA nancomposite by laser causes excitation of collective oscillations of free electrons of Ag nanoparticles. The enhancement observed in Raman spectra of the Ag-PMMA nanocomposite may be attributed to the resonance between the SPR of Ag nanoparticles and Raman spectra of PMMA [20]. The new peak at 650 $\mathrm{cm}^{-1}$ is attributed to the PVP-stablized Ag nanoparticles. A similar band was also observed in the Raman spectrum of Ag collodial solution containing about $29 \mathrm{~nm}$ particles stablized by PVP [21]. The peak at $1567 \mathrm{~cm}^{-1}$ is attributed to 


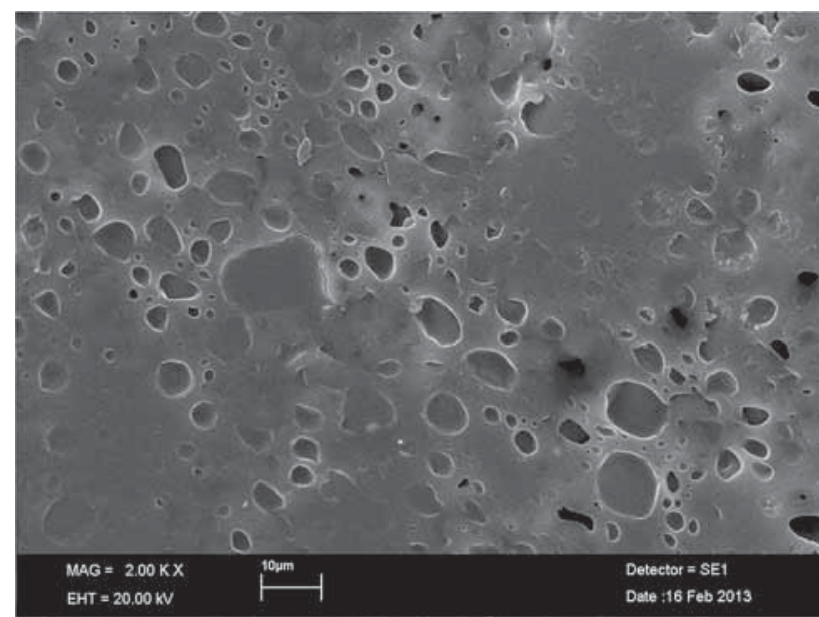

Figure 4. SEM image of Ag-PMMA nanocomposite film containing $0.039 \mathrm{wt} \% \mathrm{Ag}$ nanoparticles.

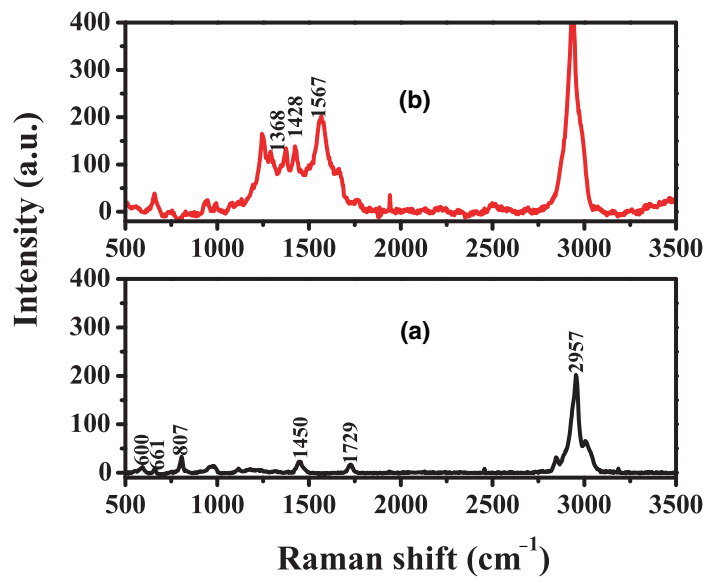

Figure 5. Raman spectra for (a) PMMA and (b) Ag (0.039 wt\%)PMMA nanocomposite film.

$(\mathrm{C}=\mathrm{C})$ stretching vibrations, at $1428 \mathrm{~cm}^{-1}$ assigns to $\mathrm{CH}_{2}$ bending vibrations, at $1372 \mathrm{~cm}^{-1}$ corresponds to disorder $\mathrm{D}$ band. The $\mathrm{D}$ band is forbidden in perfect graphite and only becomes active in the presence of disorder [22]. The shifting of peaks in Raman spectra after incorporation of Ag nanoparticles in PMMA may be due to the adsorption of polymer on the surface of Ag nanoparticles [20]. These changes in Raman spectra confirm structural modification of PMMA after incorporation of Ag nanoparticles.

Figure 6 also displays the slope of the $\log I v s . \log V$ is equal to 1 for voltage $<2 \mathrm{~V}$, which suggests that ohmic conduction is dominant mode of conduction, while at voltage $>2 \mathrm{~V}, I-V$ curves are non-linear in nature. Such non-linear behaviour of $I-V$ curves can arise from a number of non-ohmic charge transport processes such as space charge limited current (SCLC), tunnelling, Schottky and Poole-Frenkel mechanism (PF) [23]. To understand the actual conduction mechanism at voltage $>2 \mathrm{~V}, I-V$ data were plotted in the form of $\log I \mathrm{vs}$.

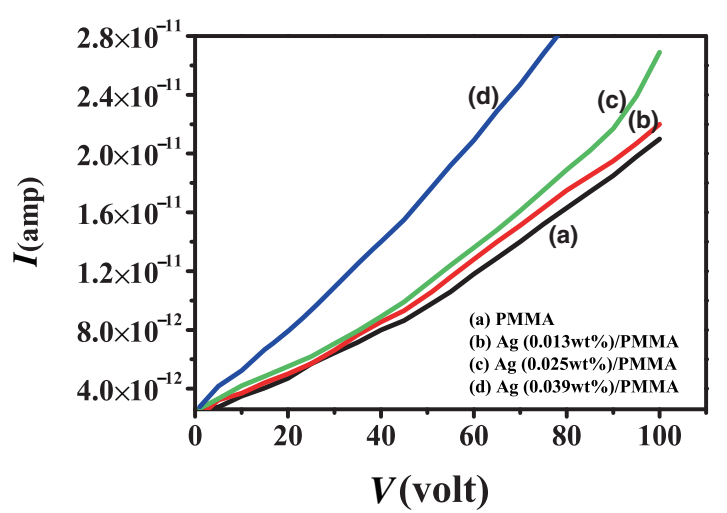

Figure 6. $I-V$ characteristics for (a) PMMA and Ag-PMMA nanocomposite films containing (b) $0.013 \mathrm{wt} \%$, (c) $0.025 \mathrm{wt} \%$ and (d) $0.039 \mathrm{wt} \% \mathrm{Ag}$ nanoparticles.

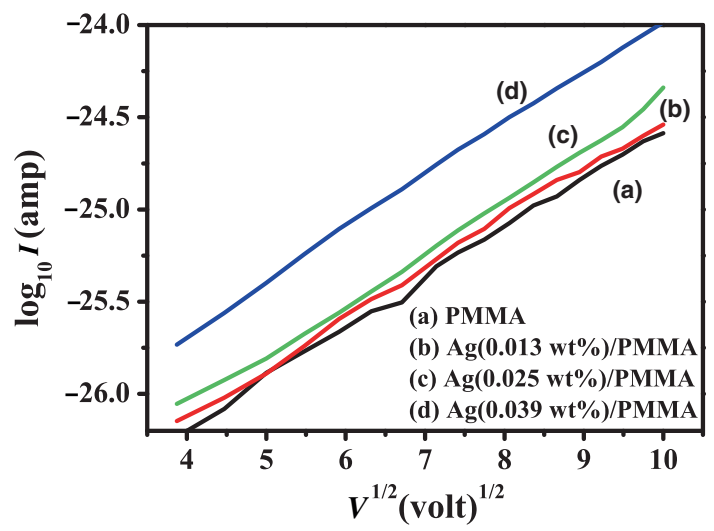

Figure 7. Plots of $\log I$ vs. $V^{1 / 2}$ for (a) PMMA and Ag-PMMA nanocomposite films containing (b) $0.013 \mathrm{wt} \%$, (c) $0.025 \mathrm{wt} \%$ and (d) $0.039 \mathrm{wt} \% \mathrm{Ag}$ nanoparticles.

$V^{1 / 2}$ (figure 7), slopes of resulting curves are compared with the equation:

$$
I \propto \exp \left(\frac{e \beta V^{1 / 2}}{K T d^{1 / 2}}\right)
$$

where $V$ is the applied voltage, $d$ the sample thickness, $e$ the electronic charge, $\beta$ the constant: characteristic of the conduction mechanism, $K$ the Boltzman's constant and $T$ the absolute temperature. The almost linear behaviour illustrated in figure 7 suggests either a Schottky or Poole-Frenkel conduction mechanism is operative in PMMA and Ag-PMMA nanocomposite films. For differentiating between these two mechanisms, the values of $\beta_{\text {exp }}$ for different nanocomposite films were obtained from the slope of $\log I v s . V^{1 / 2}$ plots using the equation:

$$
\beta_{\exp }=\frac{m K T d^{1 / 2}}{e},
$$


Table 1. Experimental and theoretical values of $\beta$ for PMMA and Ag-PMMA nanocomposite films.

\begin{tabular}{lccc}
\hline & Experimental & \multicolumn{2}{c}{ Theoretical } \\
\cline { 2 - 4 } Sample & $\beta_{\text {exp }} \times 10^{-5} \mathrm{eV}$ & $\beta_{\mathrm{S} \times 10^{-5} \mathrm{eV}}$ & $\beta_{\mathrm{PF}} \times 10^{-5} \mathrm{eV}$ \\
\hline PMMA & $\mathrm{V}^{-1 / 2} \mathrm{~m}^{1 / 2}$ & $\mathrm{~V}^{-1 / 2} \mathrm{~m}^{1 / 2}$ & $\mathrm{~V}^{-1 / 2} \mathrm{~m}^{1 / 2}$ \\
Ag (0.013 wt $\%)$-PMMA & 4.10 & - & - \\
Ag $(0.025 \mathrm{wt} \%)$-PMMA & 4.07 & 2.39 & 4.78 \\
Ag $(0.039 \mathrm{wt} \%)$-PMMA & 4.15 & - & - \\
\hline
\end{tabular}

where $m$ is the slope of $\log I v s . V^{1 / 2}$ plots for PMMA and AgPMMA nanocomposite films as shown in figure 7. Theoretical values for $\beta$ was calculated using the following relations:

For Schottky emission

$$
\beta_{\mathrm{S}}=\left(\frac{e}{4 \pi \varepsilon_{\mathrm{S}} \varepsilon_{0}}\right)^{1 / 2},
$$

and for Poole-Frenkel emission,

$$
\beta_{\mathrm{PF}}=2 \beta_{\mathrm{S}}
$$

where $\varepsilon_{0}$ is the free space permittivity and $\varepsilon_{\mathrm{s}}$ is the dielectric constant of the surrounding polymer at high frequency ( 2.5 for PMMA). The values of $\beta_{\text {exp }}$ for PMMA and Ag-PMMA nanocomposite films, along with the theoretically calculated values of $\beta_{\mathrm{S}}$ and $\beta_{\mathrm{PF}}$ are listed in table 1 . Table 1 depicts that the $\beta_{\exp }$ value is in agreement with the $\beta_{\mathrm{PF}}$, which leads to the conclusion that Poole-Frenkel mechanism is the dominating conduction mechanism in Ag-PMMA nanocomposite films. This can be attributed to a significant number of defects or traps created in PMMA after doping with Ag nanoparticles. Due to thermal activation, electrons may be emitted from traps into the conduction band of PMMA assisted by electric field and give rise to Poole-Frenkel conduction mechanism. Similar results have been reported by other authors in different nanocomposite systems. Moosvi et al [24] have fabricated $\mathrm{PTP} /\left[\mathrm{Fe}(\mathrm{CN})_{3}(\right.$ dien $\left.)\right] \cdot \mathrm{H}_{2} \mathrm{O}$ nanocomposite and reported transition of charge transport mechanism from ohmic at lower voltages to space charge limited emission at higher voltages. Mahrous et al [25] have investigated the conduction mechanism in PVC-stabilized with dibutylin laurate-maleate and reported current due to thermally generated carriers at low voltage and identified Poole-Frenkel mechanism at higher voltages.

Optical absorption spectrum is an important tool to understand band structure and electronic properties of pure- and doped polymers. The optical energy gaps have been determined by plotting the variation of $(\alpha h v)^{1 / 2} v s . h v$ (incident photon) from the first absorption edge of absorption spectra. Indirect energy gap values for PMMA are found to decrease on increasing concentration of $\mathrm{Ag}$ nanoparticles as discussed

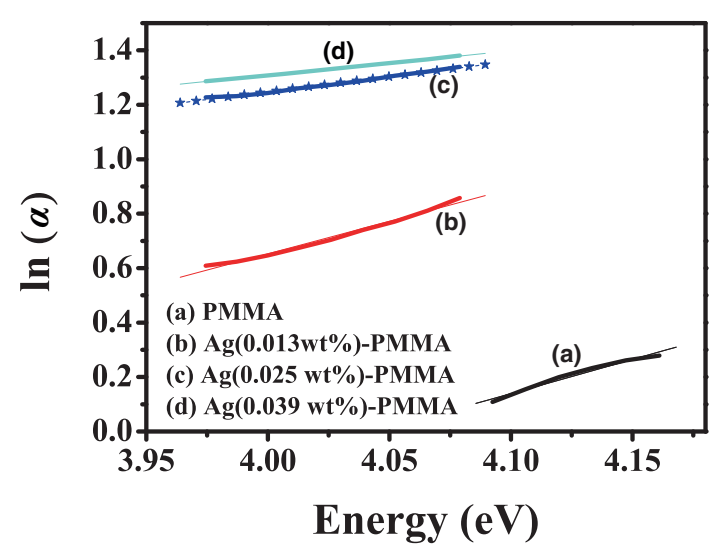

Figure 8. $\ln (\alpha)$ vs. $h v$ plots for (a) PMMA and Ag-PMMA nanocomposite films containing (b) $0.013 \mathrm{wt} \%$, (c) $0.025 \mathrm{wt} \%$ and (d) $0.039 \mathrm{wt} \% \mathrm{Ag}$ nanoparticles.

elsewhere [12]. The decrease in the optical energy gap of PMMA reflects the role of modifying the structure of PMMA by the introduction of various localized energy states within highest occupied molecular orbital (HOMO)-lowest unoccupied molecular Orbital (LUMO) gap of PMMA. Urbach's energy or Urbach's tail is the width of localized states in the optical energy gap. The extent of Urbach's tail of absorption edge can be anticipated by the relation [26]:

$$
\alpha(h v)=\alpha_{0} \exp \left(\frac{h v}{E_{\mathrm{u}}}\right)
$$

where $\alpha_{0}$ is a constant and $E_{\mathrm{u}}$ the Urbach's energy. The curves of $\ln (\alpha)$ vs. $h v$ were plotted as shown in figure 8. The reciprocal of the slopes of linear portion of the plots yield the values of $E_{\mathrm{u}}$ as shown in table 2. From table 2, it is clear that $E_{\mathrm{u}}$ increases as the concentration of $\mathrm{Ag}$ nanoparticles increases in PMMA. This increase in the $E_{\mathrm{u}}$ value points toward an increase in the width of localized states within the optical energy gap of PMMA. Due to an increase in the width of localized states, electrical conductivity of Ag-PMMA nanocomposites films should also increase with increasing concentration of $\mathrm{Ag}$ nanoparticles. Really, it is true as $I-V$ studies also show that the conductivity of PMMA increases with the increase in the concentration of $\mathrm{Ag}$ nanoparticles. 
Table 2. Urbach's energy ( $\left.E_{\mathrm{u}}\right)$ for PMMA and Ag-PMMA nanocomposite films incorporated with various concentrations of Ag nanoparticles.

\begin{tabular}{lc}
\hline Sample & $E_{\mathrm{u}}(\mathrm{eV})$ \\
\hline PMMA & 0.40 \\
Ag $(0.013 \mathrm{wt} \%)$-PMMA & 0.42 \\
Ag $(0.025 \mathrm{wt} \%)-$ PMMA & 0.91 \\
Ag $(0.039 \mathrm{wt} \%)-$ PMMA & 1.11 \\
\hline
\end{tabular}

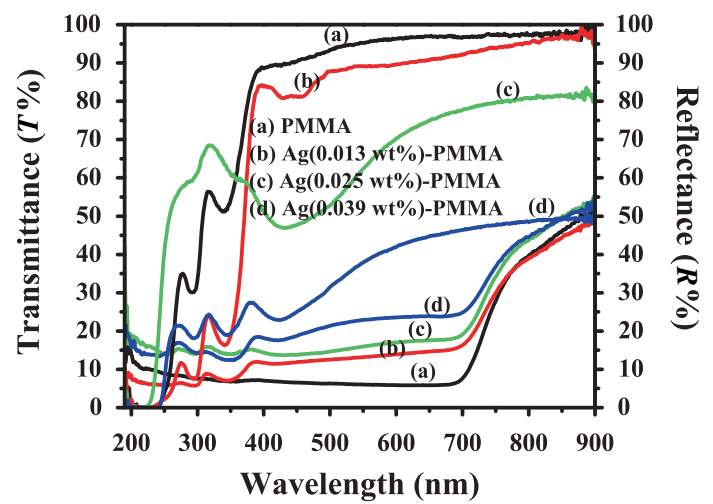

Figure 9. Transmittance $(T \%)$ and reflectance $(R \%)$ spectra for (a) PMMA and Ag-PMMA nanocomposite films containing (b) 0.013 wt $\%$, (c) $0.025 \mathrm{wt} \%$ and (d) $0.039 \mathrm{wt} \% \mathrm{Ag}$ nanoparticles.

The transmittance and reflectance of PMMA and AgPMMA nanocomposite films containing increasing concentration of Ag nanoparticles were recorded in the wavelength range of 190-900 $\mathrm{nm}$ as shown in figure 9, which depicts decrease in transmittance of PMMA from 96 to $42 \%$ measured at $600 \mathrm{~nm}$, while increase in reflectance of PMMA with incorporation of Ag nanoparticles. The dip at $\lambda \sim 422 \mathrm{~nm}$ in the transmittance spectra of Ag-PMMA nanocomposite films is attributed to the SPR of Ag nanoparticles in PMMA. This sharp fall in transmittance or high reflectance can be of potential use for non-linear optics and photonic applications such as optical switches, integrated optics, etc.

\section{Conclusions}

Ag-PMMA nanocomposite films were synthesized using $e x$ situ chemical route. The alteration in optical and structural performances of PMMA was established by UV-visible spectroscopy, TEM, SEM and Raman spectroscopy. It is observed that the concentration of Ag nanoparticles has strongly influenced the optical, structural and electrical properties of PMMA matrix. The increase in the electrical conductivity and the increase in $E_{\mathrm{u}}$ value from 0.40 to $1.11 \mathrm{eV}$ with increase in the concentration of $\mathrm{Ag}$ nanoparticles in PMMA matrix can be correlated to the formation of the localized energy states within HOMO-LUMO gap of PMMA matrix. All the nanocomposites films exhibit ohmic behaviour at voltage $<2 \mathrm{~V}$ and the Poole-Frenkel conduction mechanism at voltage region $>2 \mathrm{~V}$. This was interpreted as the emission of trapped carriers from localized states via electric perturbation.

\section{Acknowledgements}

This work was supported by Kurukshetra University, Kurukshetra. One of the authors, Alisha thanks the Haryana State Council for Science and Technology (HSCST), Panchkula, for providing the fellowship.

\section{References}

[1] Carnotenuto L N 2005 Metal-polymernanocomposites (Hoboken: Wiley) p 2

[2] Heilmann A 2003 Polymer films with embedded metal nanoparticles (Berlin: Springer)

[3] Kreibeg U and Vollmer M 1995 Optical properties of metal clusters (Springer Series in Material Science)

[4] Brandrup J, Immergut E H, Grulke E A, Abe A and Bloch D R 2005 Polymer handbook (Hoboken: Wiley)

[5] Rozra J, Saini I, Sharma A, Chandak N, Aggarwal S, Dhiman $\mathrm{R}$ et al 2012 Mater. Chem. Phys. 1341121

[6] Makvandi P, Nikfarjam N, Sharifi S N and Qazvini N T 2015 Bull. Mater. Sci. 381625

[7] Yuguang W, Tonghe Z, Andong L and Gu Z 2002 Surf. Coat. Technol. 157162

[8] Osés J, Palacio J F, Kulkarni S, Medrano A, García J A and Rodríguez R 2014 Appl. Surf. Sci. 31056

[9] Xiong Z, Wang H, Xu N, Li H, Fang B, Zhao Y et al 2015 Int. J. Hydrogen Energy 4010049

[10] Podstawka E, Ozakiand Y and Proniewicz L M 2004 Appl. Spectrosc. $\mathbf{5 8} 570$

[11] Chantal P and Eugenia K 2008 Mater. Today 1148

[12] Goyal A, Rozra J, Saini I, Sharma P K and Sharma A 2012 Adv. Mater. Res. $\mathbf{5 8 5} 134$

[13] Huang X and Sayed M 2010 J. Adv. Res. 113

[14] Mie G 1908 Ann. Phys. 330377

[15] Abyaneh K M, Paramanik D, Varma S, Gosavi S W and Kulkarni S K 2007 J. Phys. D Appl. Phys. 403771

[16] Arnold G W 1975 J. Appl. Phys. 464466

[17] Link S, Wang Z L and El-Sayed M A 1999 J. Phys. Chem. B 1033529

[18] Singho N D, Johan M R and Lah N A C 2014 Nanoscale Res. Lett. 91

[19] Pinheiro P and Fateixa S 2015 J. Raman Spectrosc. 4647

[20] Badr Y and Mahmoud A 2006 J. Appl. Polym. Sci. 993608

[21] Perkas N, Shuster M, Amirian G, Koltypin Y and Gedanken A 2008 J. Polym. Sci. Part A Polym. Chem. 461719

[22] Saini I, Sharma A, Rozra J, Dhiman R, Aggarwal S and Sharma P K 2015 J. Appl. Polym. Sci. 424641

[23] Jonscher A K 1967 Thin Solid Films 1213

[24] Moosvi S K, Majid K and Ara T 2016 Chem. Phys. 478110

[25] Mahrous S and Hanfy T A 2004 Curr. Appl. Phys. 4461

[26] Yakuphanoglua F, Barım G and Erol I 2007 Physica B 391136 